\title{
Ships Hull Corrosion Diagnosis From Close Measurements of Electric Potential in the Water
}

\author{
A. Guibert ${ }^{1,2}$, O. Chadebec ${ }^{1}$, J.-L. Coulomb ${ }^{1}$, and C. Rannou ${ }^{2}$ \\ ${ }^{1}$ G2Elab (UMR 5269 INPG-UJF-CNRS), ENSIEG, 38402 Grenoble, France \\ ${ }^{2}$ DGA/GESMA-Département DDBF, 29240 Brest Armees
}

\begin{abstract}
We present here an original method to search the corroded zones of an underwater steel structure. Nowadays, after a defined navigation period, a vessel is placed in dry dock, to examine its hull state, find the damaged areas and then paint them. This step of identification, very long and relatively inefficient, could be replaced by a series of electrical measurements which would be processed to get clues about the state of the hull. Those results are obtained thanks to the study of the cathodic protection system equipped on the hull. This new method allows a great timesaving but also a precision never reached before.
\end{abstract}

Index Terms-Boundary elements method, cathodic protection, inverse problems, regularization techniques.

\section{INTRODUCTION}

A DVANCED techniques in electrochemistry allow the effective protection of ships hull through cathodic protection. Without that, each damaged area would form with the noble parts of the hull a galvanic couple in the seawater, leading to a natural corrosion phenomenon. This reaction consists in currents circulation from the damaged areas (anodes) to the nobles parts (propellers, etc.,...). This would aggravate the state of the damaged areas with a loss of material. One solution, called sacrificial anodes cathodic protection, consists in placing metals on the hull with a more negative electrochemical potential than the structure we have to protect (some steel here). They become the new anodes of the system and are oxidized. The other solution is to distribute active anodes in noble metals (often made of platinum) on the hull. They are connected to a dc power source and will generate electric currents in the seawater, placing the damaged areas in their electrochemical immunity domain. This method, called impressed current cathodic protection (ICCP) creates currents lines and so an electromagnetic field in the seawater (see Fig. 1), measurable. The location and current densities of the anodes are known in this case.

Some mathematical methods exist to find a corrosion damage coefficient $\gamma$ of the inner parts of pipelines [1] for example or in other inner cases, based on boundary elements methods [2]. These methods start most of the time with measurements on bounds. Our study deals with extern problems, an approach not so widespread [3], predicting electromagnetic fields in the seawater created by underwater steel structure, and an original inverse method to make a diagnosis of the corrosion they encounter. More precisely, it will be based on the second cathodic protection explained before and will stand in underwater electrical measurements and their processing to find the damaged areas.

First, a forward modeling based on a boundary element method will be presented. Then, from the knowledge of the boundary conditions obtained, a calculation of electric potential anywhere in the sea water will be tackled. Finally, an

Manuscript received October 07, 2008. Current version published February 19, 2009. Corresponding author: C. Rannou (e-mail: corinne.rannou@dga.defense.gouv.fr).

Digital Object Identifier 10.1109/TMAG.2009.2012796

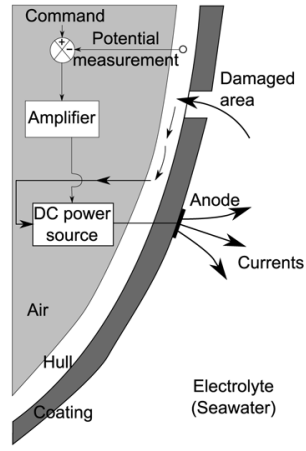

Fig. 1. Impressed current cathodic protection principle.

extrapolation of this potential calculation will be at the origin of an original inverse method. This method permits to make a diagnosis (find the unknown boundary conditions) of the hull from electric measurements in the seawater.

\section{FORWARD MODELING}

As said previously, the main goal here is to predict the electric potential in an infinite electrolyte (seawater) created by an underwater structure whose part of the boundary conditions are known. Remembering our aim is to later develop an inverse method, we choose to use a boundary element method (BEM). Indeed, inversing with a finite-elements method (FEM) is very difficult. Its other advantages are that we do not have to mesh the volumes and an infinite limit representation is not necessary.

\section{A. BEM Method in Extern Problem}

In the seawater, without additional charges, the electric potential verifies the Laplace equation

$$
\Delta \varphi=0 .
$$

Green's first identity gives

$$
\iiint_{V}(u \Delta v-v \Delta u) d V=\iiint_{S}(u(\partial v / \partial n)-v(\partial u / \partial n)) d S \text {. }
$$

Replacing $u$ by the electric potential $\varphi$ and $v$ by Green's function in 3-D

$$
G(M, P)=1 / r_{\mathrm{PM}}=1 /|\mathrm{PM}|
$$




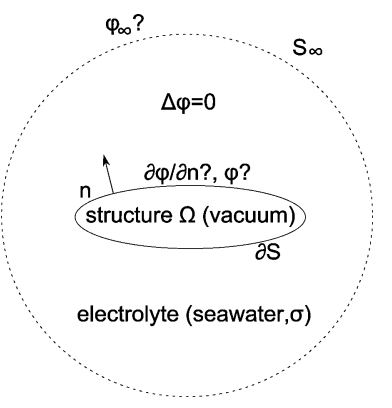

Fig. 2. Schematic representation of the boundary problem.

We obtain Green's third identity, linking the potential and its normal derivative on the whole surface

$$
\begin{aligned}
h(P) \cdot \varphi(P)=\iint_{S} \varphi(M) \cdot(\partial G(M, P) / \partial n) d S \\
\quad-\iint_{S} G(M, P) \cdot(\partial \varphi(M) / \partial n) d S
\end{aligned}
$$

where $h$ is the solid angle seen by the element of $P$. Extrapolating (4) for $P$ on the boundaries of the problem (non singular, i.e., $P$ on a plane boundary) and introducing a virtual potential for the infinity bound $\varphi_{\infty}$ (see Fig. 2), we have

$$
\begin{aligned}
-2 \pi \cdot \varphi(P) & =\iint_{S} \varphi(M) \cdot(\partial G(M, P) / \partial n) d S \\
& -\iint_{S} G(M, P) \cdot(\partial \varphi(M) / \partial n) d S-4 \pi \cdot \varphi_{\infty} .
\end{aligned}
$$

Equation (5) is applied on all the surfaces of the problem. By meshing the whole surface, making a point matching approach of our unknowns at each elements barycenter, we get the following system:

$$
\left[\begin{array}{lll}
H & T & -4 \pi
\end{array}\right] \cdot\left[\begin{array}{c}
\varphi \\
\partial \varphi / \partial n \\
\varphi_{\infty}
\end{array}\right]=0
$$

with

$$
\begin{aligned}
H_{i j} & =-\iint_{\Omega_{j}} \frac{\partial}{\partial n_{M}^{(j)}}\left(\frac{1}{r_{\mathrm{PM}}^{(i j)}}\right) d S_{M}^{j} \\
& =-\iint_{\Omega_{j}} \frac{\vec{n}_{M}^{(j)} \cdot \vec{r}_{\mathrm{PM}}^{(i j)}}{\left(r_{\mathrm{PM}}^{(i j)}\right)^{3}} d S_{M}^{j} \\
T_{i j} & =\iint_{\Omega_{j}} \frac{1}{r_{\mathrm{PM}}^{(i j)}} d S_{M}^{j} .
\end{aligned}
$$

The boundary conditions available are as follows.

- An anode region (non-polarizable electrode), whose current density $J_{A}$ is known. $\partial \varphi_{A} / \partial n$ is linked to $J_{A}$ by multiplying it by $\sigma$, the conductivity of the electrolyte.

- A cathode region (polarizable electrode), whose polarization law $\partial \varphi_{C} / \partial n=f\left(\varphi_{C}\right)$ is known and most of the time nonlinear.

- An insulator region, whose current density is null on it: $\partial \varphi_{I} / \partial n=0$.

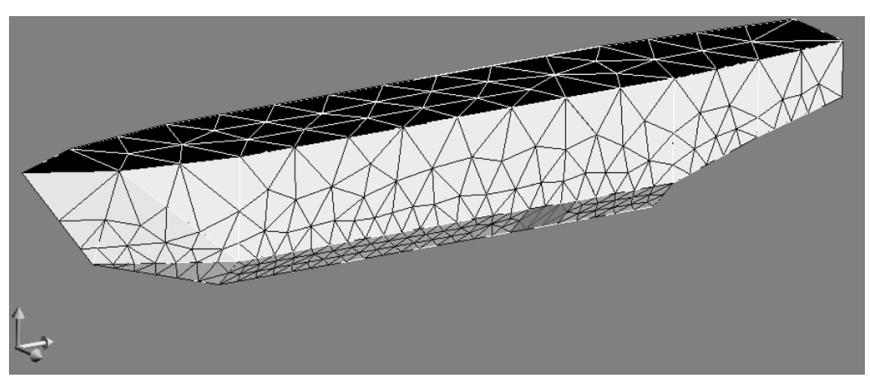

Fig. 3. Geometry meshed of an existing model.

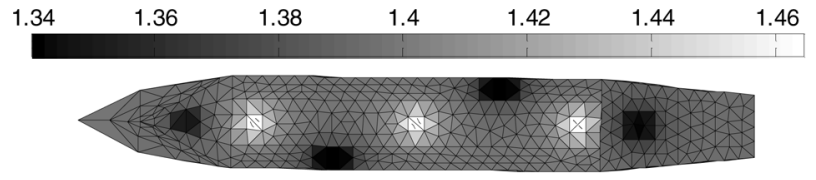

Fig. 4. Result obtained, $\varphi_{\infty}=1.3762 \mathrm{~V}$.

The system in (6) can be written by separating known and unknown variables, adding a new line (the last one) which ensures $\operatorname{div}(\mathbf{J})=0$

$$
\begin{array}{r}
{\left[\begin{array}{ccccc}
H_{11}+2 \pi & H_{12} & H_{13} & T_{12} & -4 \pi \\
H_{21} & H_{22}+2 \pi & H_{23} & T_{22} & -4 \pi \\
H_{31} & H_{32} & H_{33}+2 \pi & T_{32} & -4 \pi \\
0 & 0 & 0 & A_{2} & 0
\end{array}\right]} \\
\\
{\left[\begin{array}{c}
\varphi_{A} \\
\varphi_{C} \\
\varphi_{I} \\
\frac{\partial \varphi_{C}}{\partial n} \\
\varphi_{\infty}
\end{array}\right]=\left[\begin{array}{l}
-T_{11} \cdot \frac{\partial \varphi_{A}}{\partial n}-T_{13} \cdot \frac{\partial \varphi_{I}}{\partial n} \\
-T_{21} \cdot \frac{\partial \varphi_{A}}{\partial n}-T_{23} \cdot \frac{\partial \varphi_{I}}{\partial n} \\
-T_{31} \cdot \frac{\partial \varphi_{A}}{\partial n}-T_{33} \cdot \frac{\partial \varphi_{I}}{\partial n} \\
-A_{1} \cdot \frac{\partial \varphi_{A}}{\partial n}-A_{3} \cdot \frac{\partial \varphi_{I}}{\partial n}
\end{array}\right]}
\end{array}
$$

where $A_{i}$ is the area of the element $i$. The conditions on the insulators $\left(\partial \varphi_{I} / \partial n=0\right)$ are known. So is the polarization law linking $\partial \varphi_{c} / \partial n$ and $\varphi_{c}$, so that we can send $\varphi_{c}$ in the second member and the system becomes square. It is possible to solve it thanks to a Newton-Raphson algorithm based on the $\varphi_{c}$ variable [5].

Assumed that we have meshed the geometry in $N$ elements, the system (8) has $N+1$ equations for $N+1$ unknowns. Therefore, it can be linearly solved so that the resolution of the Newton-Raphson algorithm will be easier. The model used here is a real underwater model meshed in 1052 elements (see Fig. 3):

- 3 uniform active anodes: 6 elements;

- 4 different cathodes (damaged areas): 31 elements;

- the rest of the hull completely isolated: 1015 elements.

The result obtained with our algorithm is presented in Fig. 4, the anodes having naturally the highest potential (in white), the cathodes having the lowest ones (in black). The scale, for the whole article, is in volts.

\section{B. Electric Potential and Field Computation}

The electric potential in the electrolyte can easily be computed with (4), which amounts to make the same numerical ap- 


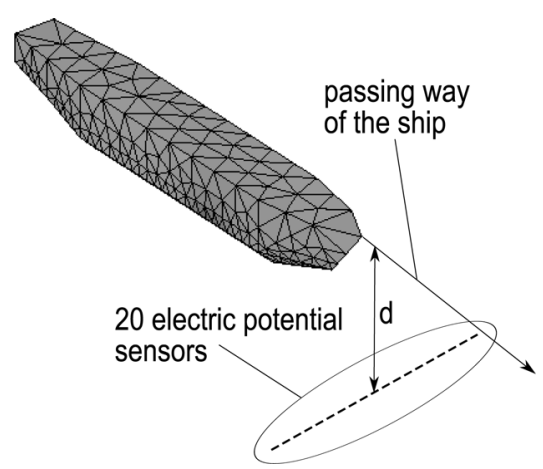

Fig. 5. Electric potential measurements principle.

proach with mesh elements (solid angle $h$ is now equal to $-4 \pi$; potential is set null in the structure)

$$
\begin{aligned}
-4 \pi \cdot \varphi(P) & =\sum_{i}^{N} \varphi\left(M_{i}\right) \iint_{\Omega_{j}} \frac{\vec{n}_{M_{i}} \cdot \vec{r}_{\mathrm{PM}_{\mathrm{i}}}}{\left(r_{\mathrm{PM}_{\mathrm{i}}}\right)^{3}} d S_{M_{i}} \\
& -\sum_{i}^{N} \frac{\partial \varphi\left(M_{i}\right)}{\partial n_{i}} \iint_{\Omega_{j}} \frac{1}{r_{\mathrm{PM}_{\mathrm{i}}}} d S_{M_{i}}-4 \pi \cdot \varphi_{\infty} .
\end{aligned}
$$

The infinite potential is not necessary here: indeed it is a virtual offset (1.3762 V in Fig. 4), so that we can remove its term: the results obtained will be centered on zero.

\section{DiAGNOSIS: USE OF AN INVERSE METHOD}

\section{A. Principle}

Contrary to the previous part, we want here to get the boundary conditions through a series of electric measurements, with only one type of data from the hull which are the current densities $\partial \varphi_{A} / \partial \mathrm{n}$ delivered by the anodes. In this paragraph, we will keep the same model example from the previous part, with 1052 elements, so that we have 2098 unknowns, noted $X$. The measurements vector will then be noted $B$ and an influence matrix A will be built so that we can write the equation

$$
A \cdot X=B \text {. }
$$

We decide to base our inverse method on electric potential measurements: indeed the electric field decreases faster in an electrolyte than the electric potential, so with potentials, the significant measurements space is bigger. Such measurements could be made by passing a ship above a perpendicular potential sensor line, as in Fig. 5.

First, the forward modeling is used to create the virtual measurements explained on Fig. 5 on a grid. Some random noise is added with maximum value equal to $5 \%$ of the maximum potential calculated. The following figure presents a potential computation from the 20 sensors shown in Fig. 5 with 70 measurement abscissas on the passing way of the ship at a 0.855 meter deep d which gives a B vector with 1400 components (see Fig. 6).

The influence matrix A contains the interactions between each mesh element and the measurements location points. It will be built with (9). With our example we have an A matrix with 1400 rows and 2098 columns which places us in an under-determined case. Solving the normal equation aims to

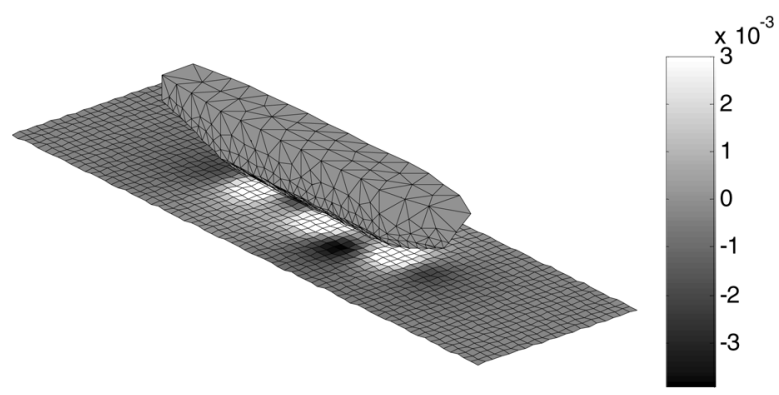

Fig. 6. Result of electric potential measurements.

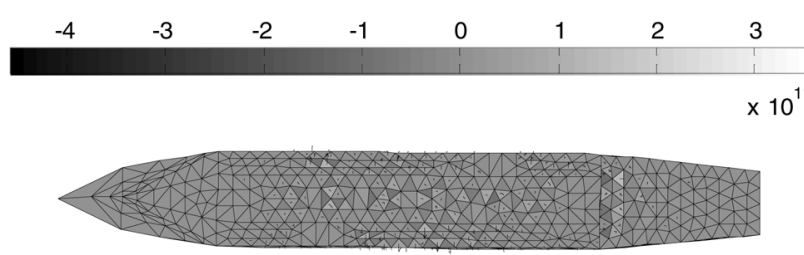

Fig. 7. Boundary conditions obtained on the hull with a direct inversion.

find $X$ that minimizes the residual norm $\|A \cdot X-B\|$. This amounts to solve $A^{t} \cdot A \cdot x=A^{t} \cdot B$. The main problem is that $A$ is most of the time very bad conditioned. Indeed, the different number of columns and rows implies a bad condition number. And more specifically the two different unknowns' types (potentials and their normal derivatives) lead to values in A with different scales. Matrix A being bad conditioned, $A^{t} \cdot A$ will be worse conditioned, giving to catastrophic results. Here, matrix A has a huge condition number equal to $4.127910^{16}$, however, we directly inverse the system (see Fig. 7).

We obtain non physic boundary conditions with maximum potential values reaching $10^{11}$ (The target values are shown in Fig. 4). We need to work on this system before inversing it to get a better solution.

\section{B. Injection of Information}

Having under-determined problems leads to inversing difficulties. A solution is to add information through other equations. A smart and original method here is to reinject Green's equations from (5). Those equations link each element to the others. It contributes to get physic compatibility between elements of the model. Indeed our problem is composed of 1052 elements so we can add 1052 equations to A (1052 lines). The problem becomes overdetermined (2452 equations for 2098 unknowns) and simpler to solve: the solution space is smartly reduced and we have to find the best one. Moreover, since it is simpler to solve an overdetermined problem, the number of measurements required can be reduced by a previous counting of our unknowns and the Green's equations we can inject.

\section{Tikhonov Regularization}

Having added Green's equations, a regularization method is necessary to choose the best solution in the space evoked before. An interesting one is the Tikhonov regularization method, which introduces a regularization matrix $\mathrm{L}$ and a regularization parameter $\lambda$. The expression to minimize is now $\|A \cdot X-B\|+$ $\lambda \cdot\|L \cdot X\|[6]$. 


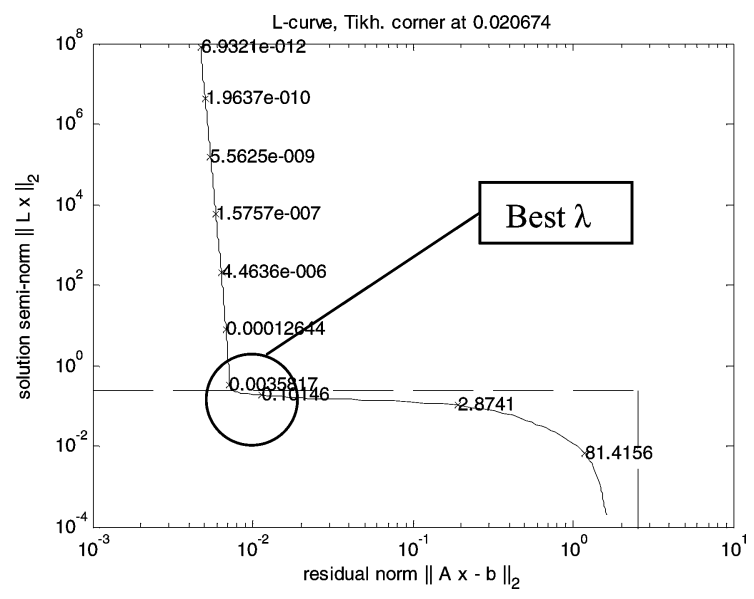

Fig. 8. Example of a L-curve with its corner.

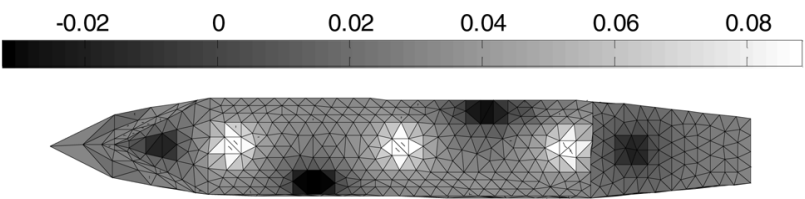

Fig. 9. Result obtained after inversion with Tikhonov regularization.

The regularization matrix $\mathrm{L}$ is square, has a dimension equal to the number of unknowns and represents the regularization type we impose. To get a minimum norm solution, $\mathrm{L}$ will be the identity matrix (order 0 ); an order 1 will favor a regular solution on the bounds... The addition of Green's equations giving a continuous solution, L matrix order chosen is 0 . Solution norm $\|L \cdot X\|$ will be the sum of the solution values, which has to be as small as possible [7].

The regularization parameter $\lambda$ is the importance given to the regularization. It defines the compromise between the residual norm $\|A \cdot X-B\|$ (precision of the solution) and the solution norm $\|L \cdot X\|$. The L-curve shown in Fig. 8, corresponding to our ship hull problem, is the graphical representation of this compromise. Its name comes from the form the curve has in many cases, meaning that the best $\lambda$ (or best compromise) stands in the corner of the L-curve. It must be mentioned that this curve can have other forms, so, according to the problem data (values of the residual norm expected), $\lambda$ choice has to be made smartly. In most of these cases $\lambda$ choice is made empirically by testing different values and observing the results.

\section{Final Result and Comparison With the Target}

Remembering the target values set in Fig. 4, the system is inversed with a $\lambda$ equal to 0.02 , corresponding to the L-Curve corner (see Fig. 8). The result obtained is shown in Fig. 9.
The darkest zones (corroded areas) correspond to the ones in Fig. 4, the method is assumed validated. It should be noticed that this inverse method algorithm do not need a virtual infinity potential to work, which explains the different offset with Fig. 4: the result is here centered in zero.

Moreover, some other methods have been tested such as the spectrum truncation of the singular value decomposition (TSVD) or the generalized cross validation (GCV) but they give less accuracy to the solution and do not permit the choice of the regularization order (for the TSVD).

\section{CONCLUSION}

We have presented here an original method to make a diagnosis of ships hull corrosion from electric measurements in the electrolyte and not on the bounds. We have seen that, through Green's equations, a few data and an appropriate series of electric potential measurements, we can get clues and even precise locations of damaged areas on the hull. Those results are obtained with efficiency and a great time saving, compared to current methods. To validate our numerical processing, a parallelepipedic bowl in PMMA (Plexiglas) has been built with a system of electric potential measurement on it. Models can be flown in it such as some painted steel with damaged areas and an appropriate cathodic protection on it. Electric potential measurements on strategic points in the bowl permit the validation of the presented algorithms, imposing current densities injected through a PC with appropriate software and an electrolyte whose salinity is controlled. These tests are in work, giving matching results and will be presented in future articles. It should be noticed that not only ships could get advantages from this method, but also pipelines, oil rigs, or any underwater steel structure.

\section{REFERENCES}

[1] J. Cheng, M. Choulli, and X. Yang, "An iterative BEM for the inverse problem of detecting corrosion in a pipe," Frontiers Prospects Contemporary Applied Mathematics, vol. 6, pp. 1-17, 2006.

[2] C. A. Brebbia, J. C. F. Telles, and L. C. Wrobel, Boundary Elements Techniques, Theory and Applications in Engineering. Berlin, Germany: Springer-Verlag, 1984.

[3] R. Adey and J. M. W. Baynham, Predicting Corrosion Related Magnetics. Southampton, U.K.: BEASY communication, 2000.

[4] N. G. Zamani, J. M. Chuang, and J. F. Porter, "BEM simulation of cathodic protection systems employed in infinite electrolytes," Int. J. for Numer. Methods Eng., vol. 24, pp. 605-620, 1987.

[5] G. Dhatt, G. Touzot, and E. Lefrançois, "Méthode Des Éléments Finis," 2007, pp. 404-426.

[6] C. Hansen, "Rank-deficient and discrete ill-posed problems, numerical aspects of linear inversion," 1998.

[7] O. Chadebec, J. L. Coulomb, J. P. Bongiraud, G. Cauffet, and P. Le Thiec, "Recent improvements for solving inverse magnetostatic problem applied to thin hulls," IEEE Trans. Magn., vol. 38, no. 2, pp. 1005-1008, Mar. 2002. 